\title{
The Role of Quality Control Circles in Sustained Improment of Nurses, Handover in Emergency Department
}

\author{
Xie Shen ${ }^{1 \#}$, Yueguang Dai ${ }^{24}$, Lulu Xiu ${ }^{3}$, Lei Wang ${ }^{2}$, Zhijie $\mathrm{Hu}^{2}$, Lingyu $\mathrm{Li}^{4}$, Xia $\mathrm{Liu}^{2^{*}}$ \\ ${ }^{1}$ Department of Emergency, Affiliated Hospital of Qingdao University, Qingdao266000, Shandong, P.R, China. \\ ${ }^{2}$ Department of Neurosurgery, Affiliated Hospital of Qingdao University, Qingdao266000, Shandong, P.R, China. \\ ${ }^{3}$ Department of Respiratory, Affiliated Hospital of Qingdao University, Qingdao266000, Shandong, P.R, China. \\ ${ }^{4}$ Department of Cardiology, Affiliated Hospital of Qingdao University, Qingdao266000, Shandong, P.R, China.
}

\begin{abstract}
Article Details
Article Type: Research Article

Received date: $30^{\text {th }}$ August, 2020

Accepted date: $15^{\text {th }}$ October, 2020

Published date: $19^{\text {th }}$ October, 2020

"Corresponding Author: Xia Liu, Department of Neurosurgery, Affiliated Hospital of Qingdao University, Qingdao266000, Shandong, P.R, China. E-mail: 1xqdfsyy@126.com

\#Co-First Author: Xie Shen and Yueguang Dai Chen, Affiliated Hospital of Qingdao University, Qingdao266000, Shandong, P.R, China.

Citation: Shen, X., Dai, Y., Xiu, L., Wang, 1., Hu, Z., Li, L., \& Liu, X. (2020). The role of quality control circles in sustained improment of nurses, handover in emergency department. J Comp Nurs Res Care 5(2):164. doi: https://doi.org/10.33790/ jenrc1100164.

Copyright: (C2020, This is an open-access article distributed under the terms of the Creative Commons Attribution License 4.0, which permits unrestricted use, distribution, and reproduction in any medium, provided the original author and source are credited.
\end{abstract}

\begin{abstract}
Purpose: Compared to common departments, the handover defects in the emergency department are continuously increasing due to its environment and complexity. In this study, the quality control circles activity is carried out to lower the handover defect rate in the emergency department.

Method: The list is checked to determine the handover defect rate as $13.8 \%$. In order to lower the nurses handover defect in the emergency department, we are planning to carry out the quality control circles activities and adopt the model plan-do-check-action (PDCA) in next 12 months.
\end{abstract}

Results: In terms of PDCA circle, nurses handover defect rate are lowered to $5.5 \%$ from original $13.8 \%$ in the emergency department. The handover quality has been obviously improved, nurses' sense of professional value and the patients' degree of satisfaction has been obviously improved as well.

Conclusion: The handover defect rate can be lowered by QCC in the emergency department, improve health care quality and secure patients' safety.

Keywords: The Quality Control Circles Nurses Handover

\section{Introduction}

Handover is a process to hand over patients' information, the care plan and care responsibilities between patient caregivers. Unclear handover will give rise to adverse impact on continuous care for patients [1-2]. Patients in the emergency department are characterized by critical and complex conditions, large fluidity, numerous treatment and care items. In this case, oral handover is easy to bring about a loss of information, information inconsistency or even error and other problems, which will affect patients' subsequent care and recovery. The study demonstrates that, the handover defect may lead to higher hospitalization expenses and lower patients satisfactions, and trigger longer handover time, More adverse events will be caused there from [3-5].

The quality control circle (QCC) follows the process "plan-docheck-action", which is a comprehensive and scientific management mode and has taken excellent effect in the medical field[5]. In this study, a QCC was established to lower the nurses handover defect rate in the emergency department, which not only improved our handover defect rate, but also promoted the application and practice of quality management and control tools.

\section{Methods}

Determine the theme of QCC

A QCC team named as "Emergency ROOM" has been established to deliver the concept of "heal the wounded and save the dying, no delay is allowed" the quality control circles contain totally 8 members, including two head nurses in the emergency department, 6 nurses in the emergency department. Director of Nursing Department who is responsible for nursing quality acts as the instructor for this activity. In this activity, a QCC meeting will be held once each month to assess, feedback the performance of each team member, conclude experiences and shortage, amend the nursing measures, so as to realize the continuous improvement of quality.

The theme of QCC we determined is "Lower the nurses handover defect rate in the emergency department". The main causes are as follows, first of all, the handover defect rate obtained in domestic investigation is $17.4 \% \sim 60.30 \%[6-8]$. Secondly, patients in the emergency department are characterized by large quantity, serious conditions and large fluidity. Thirdly, nurses in our department are characterized by their young age, low level and large fluidity.

\section{Propose activity plan}

According to the process and theme of QCC, activities and duration of each member to implement each step under the plan will be mapped to a Gantt chart. Overall plan of the activity is approved by the nursing department and executed during August 2017 to February 2019.

\section{Current situation investigation and target setting}

Through sorting the process, the bedside handover part is determined as the critical link of improvement. Members of quality control circles team prepare the check list (list 1), five members are in charge of following a nurse's check during nurses' bedside handover. 
Before check, personnel who are in charge of check will accept unified training to secure same quality of check.There are 63 nurses, who have been working in the emergency department for more than one year, are in charge of examination. There are 4131 inspection items in total, with a defect rate of $13.8 \%$. Through the Pareto drawn, the form illustrates that: The handover defects mainly reflect at two aspects: patients information handover and catheter handover. In view of this basic goal is set, the ability to circle is evaluated, and then the goal value is calculated by formula. The formula is current situation value-improvement value $=$ current situation value-(current situation value $*$ important points of improvement $*$ circle ability $)=$ $13.8 \%-13.8 \% \times 81.1 \% \times 65 \%=6.5 \%$.

\section{Causes analysis and countermeasures making}

The fish bone diagram is drawn from four aspects including personnel, environment, system and method by using the brainstorming method (figure 1). The potential capacity caused by the principle of "5,3,1" is graded and five main reasons are selected by the method of 80/20: 1 . Patient information is not familiar 2 . Information transmitted error3. Numerous items to be handed over 4. Insufficient training 5. Nurses lack experiences and qualification. For the five causes, a follow-up check list is made and drew a pareto and three root causes are identified finally. For the three root causes, several countermeasures are formulated by using the brainstorming method. Finally the countermeasures are developed through integration and sorted by the principle of $5 \mathrm{~W} 1 \mathrm{H}$, namely: (1) the overall care of the responsible team is implemented in the emergency department (2) formulate handover control list (3) SBAR standardized communication.

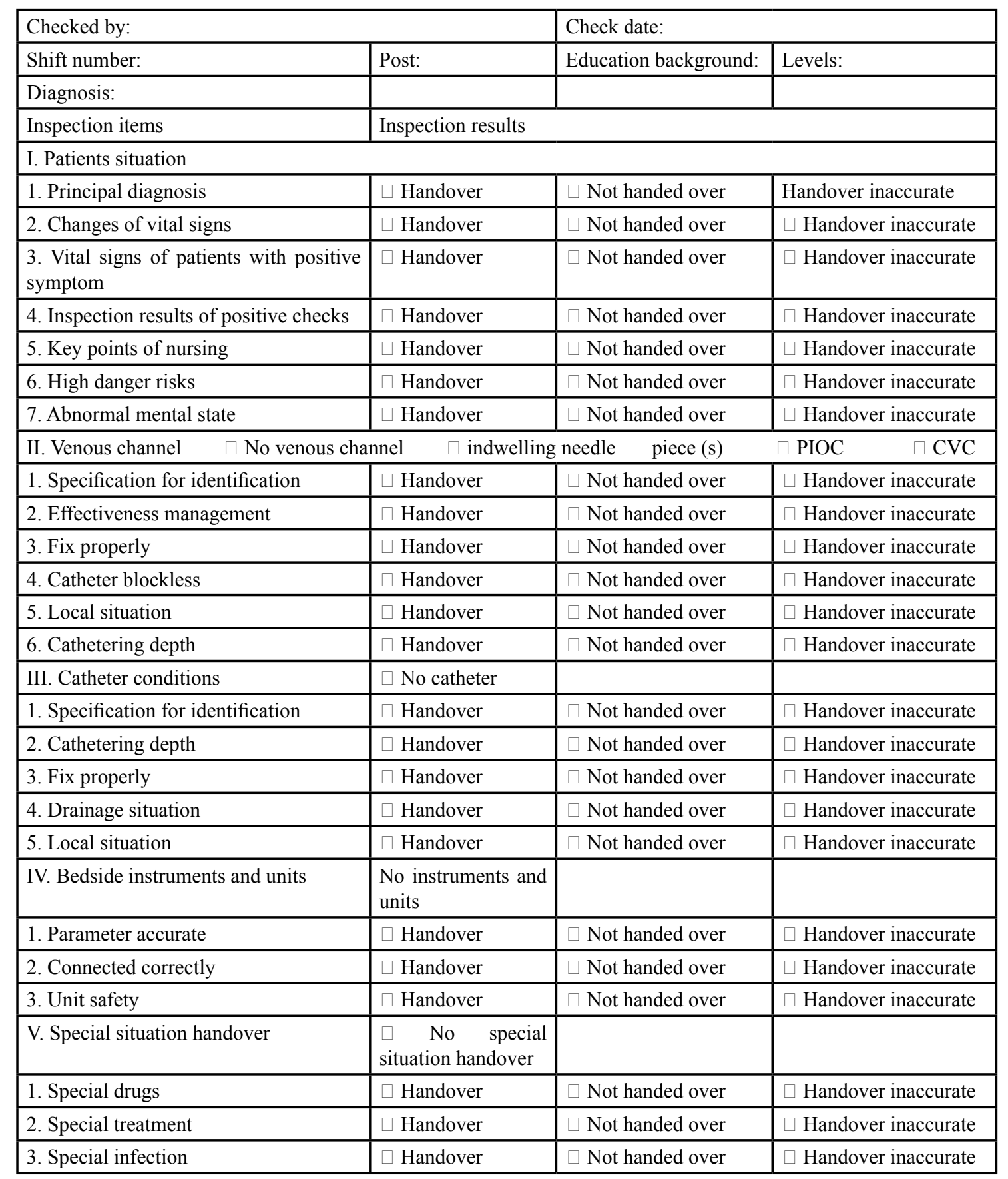

Table 1 Emergency Department Nurses Handover Defect Rate Check List 


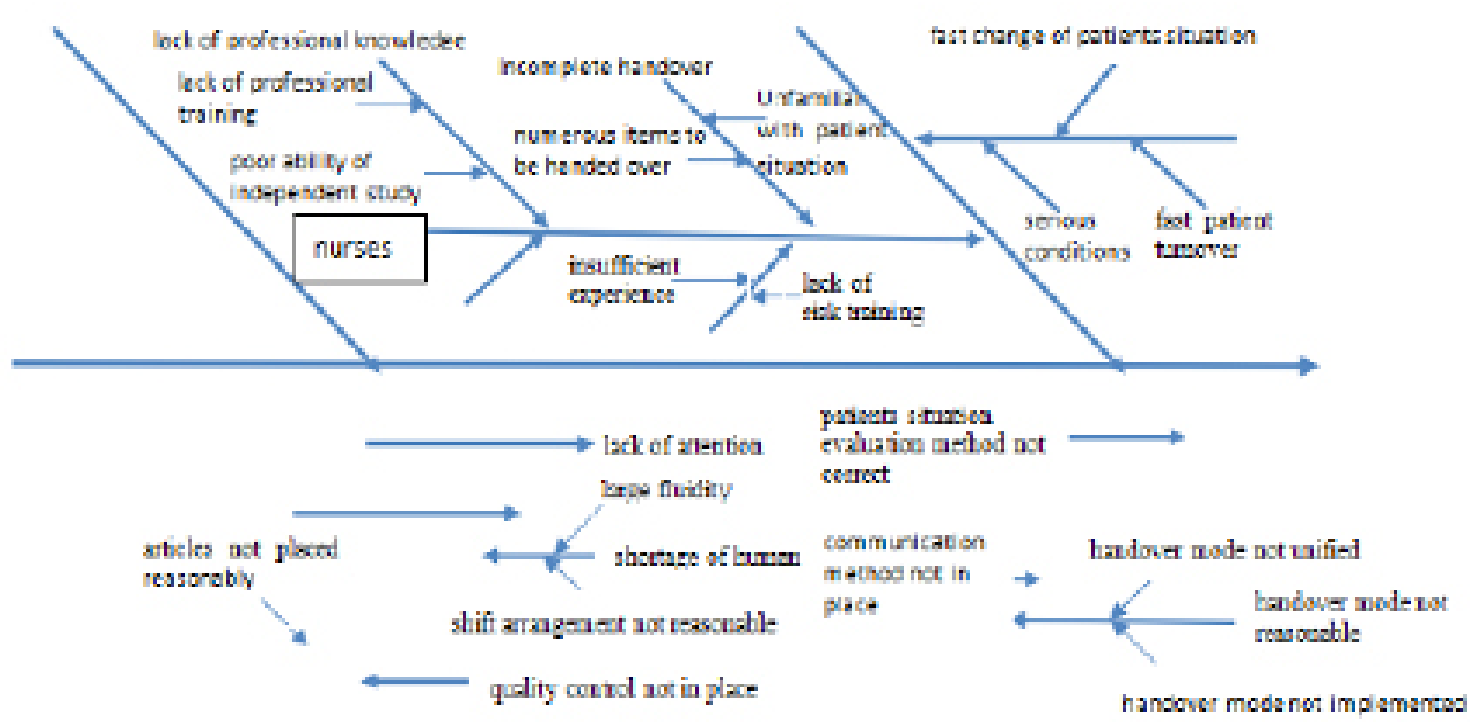

Figure 1 Emergency Department Nurses Handover Defect Causes Analys

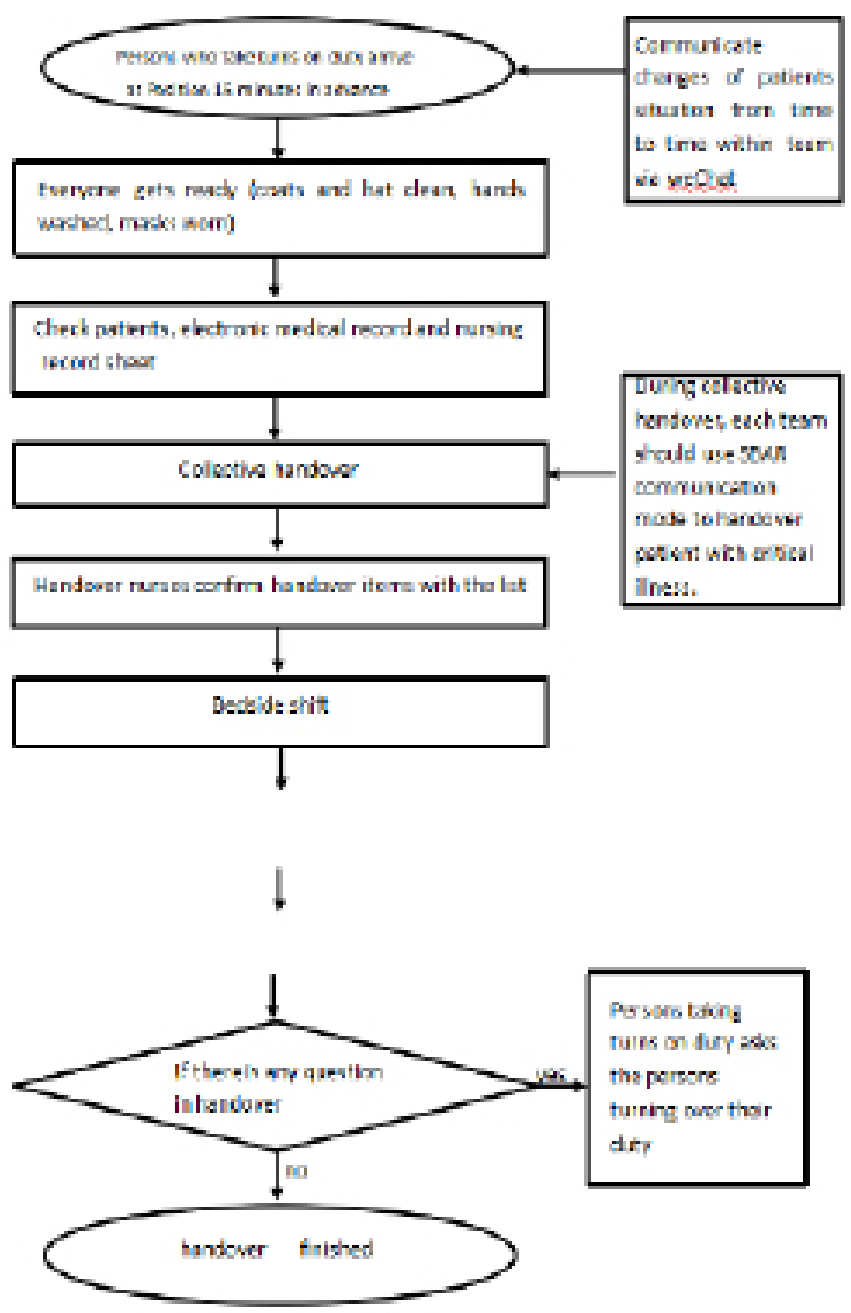

Figure. 2 Emergency Department Nurses Handover Standardized Process 


\section{Countermeasure implementation}

Firstly, the overall management of the responsible team is implemented. According to the matching and level of human resources, the structure of the team responsibilities is optimized, and responsible team for holistic care is implemented as a unit. Head nurses select team members according to experiences and qualifications. Each team is limited to 8-10 team members; the patient's condition is communicated in real time and dynamically by the person on duty every day through WeChat. Then the handover list is formulated. Next, the preliminary draft of handover list is formulated on the basis of summarizing experiences, consulting literature and the semi-structured interview. Then an emergency department nurses handover list is developed through two rounds of experts enquiry. After which, the relevant training is carried out according to the content of the list, and employed it clinically. Thirdly, the SBAR communication mode is used during patients situation handover. In the course of the morning collective handover in the department, the situation of key patients is transferred through SBAR mode. Single disease SBAR communication mode was developed. During the bedside handover process, SBAR mode is used to hand over the patients situation. SBAR communication handover is as the last station in OSCE assessment.

\section{Inspection}

After the activity, the same check list is used to calculate the handover defect rate again. The Chinese version nurses handover quality evaluation scale [9-10] is used to evaluate the handover quality from eight aspects of QCC team's ability, which containing Cohesion, ability to solve problems, learning initiative, sense of honor, quality control measures, sense of achievability.

Investigators collect the problems and suggestions of nurses in the evaluation team and clinical nurses during bedside handover process via focus group discussion, to amend and perfect the handover process and standardize it (see Figure 2), and apply it into practice on a yearly basis.

\section{Statistical analysis}

Quantitative data are presented as mean \pm standard deviation (SD) by using SPSS21.0 software, An unpaired student's test at two sides or one-way variance analysis is used to carry out statistical analysis. A P value of $<0.05$ was considered statistically significant.

\section{Results}

In this study, after establishing QCC, the patient information handover defect rate in the emergency department drops to $5.5 \%$ from original $13.8 \%$. The awareness rate of nurses on patients' situation is greatly improved $(\mathrm{P}<0.05)$ (Table 2$)$. The scores between handover quality evaluation and each dimension are statistically significant $(\mathrm{P}<0.05)$ (Table 3).

Meanwhile, the cohesion, learning initiative, ability to find and solve problems are greatly improved.

\begin{tabular}{|l|l|l|l|l|}
\hline Dimension & Before QCC & After QCC & $\mathrm{t}$ & $\mathrm{p}$ \\
\hline Information quality & $27.48 \pm 2.13$ & $30.36 \pm 2.68$ & -7.314 & 0.000 \\
\hline Interaction support & $17.47 \pm 1.81$ & $19.39 \pm 2.60$ & -5.271 & 0.000 \\
\hline Efficiency & $15.40 \pm 1.66$ & $16.58 \pm 1.37$ & -7.784 & 0.000 \\
\hline Total score & $60.39 \pm 3.22$ & $67.73 \pm 3.09$ & -14.377 & 0.000 \\
\hline
\end{tabular}

Table. 2 Comparison of handover quality scores before and after implementation of measures

\section{Discussion}

The investigation of Agency for Healthcare Research and Quality's (AHRQ) shows that, $49 \%$ medical staff are negligent in handover work by missing patients information. In this study, after establishing QCC, the patient information handover defect rate in the emergency department dropped to $5.5 \%$ from the original $13.8 \%$. This study demonstrating that, QCC is exceedingly effective in lowering nurses handover defect rate and improving the handover quality. Information continuity is ensured through the overall management of the responsible team. By relying on the We Chat platform, nurses can learn about patients' situation even if not on duty. There are many projects to be transferred for patients observed in the emergency room. The list enumerates the key points of handover shifts to avoid missing and reduce the occurrence of information missing due to memory or interruption events [11-12]. SBAR communication can realize clearer and complete handover, highlight the key points and make it easier for nurses to receive information in shifts [13-14]. In this process, the nurse's ability to think independently has been improved. Through the knowledge learned, the ability to analyze and summarize is also strengthened. The patient information can be clearly mastered, and the main question of patients can be precisely determined, so that the content delivered by nurses is more appropriate, so as to provide timely and safe medical services for patients.

Nursing quality depends not only on the effectiveness of nursing quality management methods, but also relies on nurses' quality awareness and participation in quality monitoring organization. In this study, the handover quality is checked monthly by four circle members under the quality control method and record it in the handover report. After random inspection, the feedback information is collected in time by circle members through communication and discussion with handover nurses. The implementation of measures should be constantly improved to ensure the effectiveness and improve the quality of handover.

Conflicts of interest: Authors report no conflict or competing interest.

\section{References}

1. Lautz, A.J., Martin, K.C., Nishisaki, A. et al. (2018). Focused Training for the handover of critical patient information during simulated pediatric emergencies[J]. Hosp Pediatr, 8(4):227-231.

2. Ferorelli, D., Giandola, T., Laterza, M. et al. (2017). Handover checklist:testing a standardization process in an Italian hospital. Risk Manag Healthc Policy, 10:87-93.

3. Klim, S., Kelly, A.M., Kerr, D., et al. (2010). Developing a framework for nursing handover in the emergency department: an individualised and systematic approach [J]. Journal of Clinnical nursing, 22, 2233-2243.

4. Liu, X., Chen, H.Y., Huang, R.,et al. (2016). Application of handover among nurse based on I-PASS condition report mode for CICU patient. Journal of Nursing Science, 31(15):13-16.

5. Chen, P., Yuan, T., Sun, Q.F., et al. (2016). Role of quality control circle in sustained improvement of hand hygiene compliance:an observational study in a stomatology hospital in Shandong, China[J]. Antimicrob Resist Infect Control. 5:54.

6. Agency for Health care Research and Quality. (2016). Hospital survey on patients safety culture :2016 user comparative database report $[\mathrm{R}]$. 
6. Agency for Health care Research and Quality. (2016). Hospital survey on patients safety culture :2016 user comparative database report $[\mathrm{R}]$.

7. Redley, B., Botti, M., Wood, B., et al. (2017). Interprofessional communication supporting clinical handover in emergency departments: An observation study[J]. Australasian Emergency Nursing Journal Aenj, 20(3).

8. Song, X.C., Huang, P.Y., Xu, H., et al. (2017). Effect of quality control circle activities on reducing the shift handover defect rate of emergency department nurse[J]. Journal of Nursing Education, 32(5):413-415.

9. O'Connell, B., Oekerby, C., \& Hawkins, M. (2014). Construct validity and reliability of the Handover Evaluation Scale[J]. $J$ Clin Nurs 23(3/4) 560-570.

10. LIU, J., LIU, Yan-hui., \& YANG, Ji-peng. (2015). Reliability and validity of the Chinese version of Handover Evaluation Scale[J]. Chinese Journal of Nursing, 50(5):552-555.
11. Scott, S.S., Henneman, E.A., Nathanson, B.H., et al. (2020). Use of a Transfusion Checklist by Student Nurses to Improve Patient Safety[J]. J Nurse Prof Dev, 36(1):33-38.

12. Sharp, L., Dahlen, C., Bergenmar, M., et al. (2019). Use of a transfusion checklist by student nurses to improve patient safety[J]. Scand J Caring Sci, 33(4):892-901.

13. Abbaszade, A., Assarroudi, A., Armat, M.R., et al. (2020). Evaluation of the Impact of Handoff Based on the SBAR Technique on Quality of Nursing Care[J]. J Nurs Care Qual.

14. Uhm, J.Y., Ko, Y., \& Kim, S. (2019), Implementation of an SBAR communication program based on experiential learning theory in a pediatric nursing practicum: A quasi-experimental study. Nurse Educ Today, 80:78-84. 